\title{
Functional, Clinical, and Sociodemographic Variables Associated with Risk of In-Hospital Mortality by COVID-19 in People over 80 Years Old
}

\author{
I. Rodríguez-Sánchez ${ }^{l}$, M. Redondo-Martinn ${ }^{l}$, L. Furones-Fernández ${ }^{l}$, M. Méndez-Hinojosa ${ }^{l}$, Á. Chen-Chim ${ }^{l}$, \\ R. Saavedra-Palacios ${ }^{1}$, P. Gil-Gregorio ${ }^{1,2}$
}

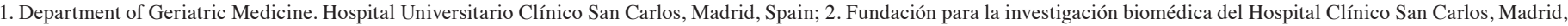
Spain

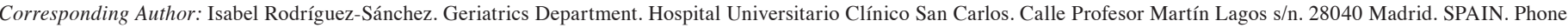
number: (+34) 91 3303000. Email: isabelrs89@gmail.com

\begin{abstract}
OBJECTIVES: The objective is to assess the role of functional, clinical, and analytic parameters in predicting mortality in older patients hospitalized due to COVID-19.

DESIGN: Cohort study with a mean follow-up of 12.8 days.

SETTING: Public university hospital (Madrid, Spain).

PARTICIPANTS: 499 patients 80 and above consecutively admitted to a Spanish public university hospital between 4 March 2020 and 16 May 2020.

MEASUREMENTS: Mortality was the main outcome. Data of sociodemographic variables (age, sex, living), comorbidities, polypharmacy, functional status, date of hospital admission and length of stay was recorded. Clinical symptoms, laboratory and X-ray findings were collected at time of admission. For multivariant analysis, logistic regressions were performed to identify risk factors for death.

RESULTS: Mean age was $86.7 \pm 4.4$ with $37 \%$ of death. Mortality was associated with male gender [odds ratio (OR) 1.50; $95 \%$ confidence interval (CI) 1.01-2.24], with a 5-points increase on Barthel Index [OR 1.01 (95\%CI 1.00-1.02)], higher Charlson Index score [OR 1.13 (95\%CI 1.02-1.26)] and comorbidities [OR 1.28 (95\%CI 1.061.53)], hyperpolipharmacy [OR 2.00 (95\% CI 1.04-3.82)], unilateral pneumonia [OR 1.83 (95\% CI 1.01-3.30)], higher levels of C-reactive protein [OR $1.09(95 \% \mathrm{CI} 1.06-1.12)$ ] and creatine [OR 1.48 (95\% CI 1.15-1.89)]. Higher oxygen levels were a protective factor [OR 0.92 (95\% CI 0.89-0.95)].

CONCLUSIONS: Functional status, being male, a higher burden of comorbidities, hyperpolipharmacy, unilateral pneumonia and some laboratory parameters predict in-hospital mortality in this older population. The knowledge of these mortality risk factors should be used to improve the survival of older hospitalized patients.
\end{abstract}

Key words: SARS-Cov-2, functional status, blood parameters, mortality, older people.

\section{Introduction}

T The onset and appearance of SARS COV2 virus and its disease begins in China, Wuhan on December 2019 (1). According to the World Health Organization (WHO) epidemiological data until February 2021, reports more than 110 million confirmed cases and more than 2 million deaths, with the United States and Europe leading that list (2). There is strong and sufficient evidence to determine that older adults and specifically the $\geq 80$-year-old group, are especially susceptible to SARS COV2 infection, causing a spectrum of more severe clinical manifestations $(3,4)$ leading to an elevated rate of mortality, being five times higher than the global average for those younger than 80 years old (5). Although the cases during the first wave in Spain (February 15th to May 31 st, 2020) in people $\geq 80$ represents $23.4 \%$ (6), this population accounted for $64.7 \%$ of the total mortality (7).

The symptoms of this disease include from mild or asymptomatic forms in a large part of the cases, to moderate or severe respiratory syndrome, where the inflammatory response plays an important role and, in many cases, triggers a cytochemical storm (8). Additionally, their vulnerability to adverse outcomes is increased due to their higher burden of comorbidities, increasing with age and rising to approximately $15 \%$ or higher (5). Moreover, some biomarkers have also been established as risk factors for mortality in older population infected by SARS-Cov-2 such as proinflamatory parameters (e.g IL-1, IL-6, or C-reactive protein) $(4,9,10)$ lymphopenia or thrombopenia (11), or nutritional status assessed by levels of albumin $(4,12)$. All these factors, in addition to some others like it is the case of functional status $(3,13,14)$ can help to explain the poor prognosis observed in older populations and the differences in the risk profile between older and adult populations.

However, very few studies have been focused on the segment of the older population with the poorest prognosis, that is the group older than 80 , as previously mentioned. The aim of this study is to describe the mortality in the oldest-old people hospitalized due to SARS-Cov-2 and to assess the factors involved in the risk of death, including comorbidities, functional status, clinical, laboratory and X-ray findings.

\section{Methods}

\section{Study design and participants}

We performed a cohort monocentric study at a public 861beds University Hospital in Madrid (Spain), including all patients aged 80 years-old and older consecutively admitted since 4 March 2020 to 16 May 2020 during the first wave of pandemic. In all of them, diagnosis of infection for SARS- 
Table 1. Sociodemographic factors, functional status, chronic morbidities and hospital mortality in old patients hospitalized by COVID-19 [n=499]

\begin{tabular}{|c|c|c|c|c|}
\hline Characteristics & Total & Recovered $(n=319 ; 63 \%)$ & Death $(n=187 ; 37 \%)$ & P value \\
\hline Age; mean (SD) & $86.7(4.4)$ & $86.5(4.2)$ & $87.1(4.7)$ & 0.06 \\
\hline Male; n (\%) & $245(49.1)$ & $143(45.4)$ & $102(55.4)$ & 0.03 \\
\hline Living; $\mathrm{n}(\%)$ & & & & 0.06 \\
\hline Community-dwelling & $421(84.4)$ & $273(86.7)$ & $148(80.4)$ & \\
\hline Barthel Index; mean (SD) & $78.1(27.5)$ & $80.5(25.7)$ & $73.8(29.9)$ & 0.02 \\
\hline Hypertension; $\mathrm{n}(\%)$ & $406(81.4)$ & $252(80.0)$ & $154(83.7)$ & 0.31 \\
\hline Diabetes; $\mathrm{n}(\%)$ & $146(29.3)$ & $81(25.7)$ & $65(36.3)$ & 0.02 \\
\hline Chronic obstructive respiratory disease; $\mathrm{n}(\%)$ & $90(18.0)$ & $54(17.1)$ & $36(19.6)$ & 0.50 \\
\hline Dementia; n (\%) & $124(24.9)$ & $67(21.3)$ & $57(31.0)$ & 0.02 \\
\hline Chronic renal failure; $\mathrm{n}(\%)$ & $100(20.0)$ & $58(18.4)$ & $42(22.8)$ & 0.24 \\
\hline Cerebrovascular disease; $\mathrm{n}(\%)$ & $57(11.4)$ & $28(8.9)$ & $29(15.8)$ & 0.02 \\
\hline Cancer; n (\%) & $102(20.4)$ & $62(19.7)$ & $40(21.7)$ & 0.58 \\
\hline CIRSG score; mean (SD) & $11.3(5.6)$ & $10.9(5.3)$ & $11.9(6.1)$ & 0.04 \\
\hline Charlson score; mean (SD) & $5.9(1.9)$ & $5.7(1.6)$ & $6.3(2.3)$ & $<0.001$ \\
\hline Polypharmacy; n (\%) & & & & 0.03 \\
\hline No $(<5)$ & $136(27.3)$ & $98(31.1)$ & $38(20.7)$ & \\
\hline Yes $(5-9)$ & $200(40.1)$ & $124(39.4)$ & $76(41.3)$ & \\
\hline
\end{tabular}

SD: Standard Deviation; \%: Percentage; *p-values for differences in means were obtained from ANOVA or Kruskall Wallis, as appropriate; while p-values for differences in proportions were obtained from chi squared tests

CoV-2 was confirmed at admission through a positive result on polymerase chain reaction test of a nasopharyngeal sample. The initial sample size was 538. Participants with no information on Barthel Index, usual medication, and levels of C-reactive protein (CRP), nor renal function (creatine) were excluded. Thus, the final sample for analysis was composed by 499 subjects (Figure 1). There were no differences according to age, gender and comorbidities between those excluded and the final sample.

\section{Study variables}

\section{Outcomes}

Hospital mortality was the main outcome of this study. Patients were followed until hospital discharge or in-hospital death, whichever was first.

\section{Predictors and adjusting variables}

Sociodemographic variables: data on age, gender, and living (community-dwelling or nursing homes) were collected from clinical records.

Functional status: The functional status was based on Barthel Index obtained from both clinical records or primary health care records.

Comorbidities and usual medication: information on comorbidities before admission (hypertension, diabetes, chronic obstructive pulmonary disease, chronic heart failure, ischemic cardiopathy, dementia, chronic renal failure, cerebrovascular disease, cancer, and tuberculosis) was also obtained. Stemming from this information we calculated the Charlson index and the Cumulative Illness Rating Scale-Geriatric (CIRS-G). Polypharmacy was assessed according to the number of usual drug treatment. Patients were classified in 3 groups: without polypharmacy (<5 drugs), polypharmacy (5-9 drugs), and hyperpolipharmacy $(\geq 10)$.

Clinical manifestations: symptoms at admission (fever, cough, dyspnea, oxygen saturation, anorexia, myalgias or arthralgias, headache, digestive symptoms, dizziness, and ageusia/anosmia) were also collected.

Laboratory and imaging findings: data on initial laboratory testing was defined as test results obtained within the first 24 hours at the Emergency department (ED). The diagnosis of pneumonia on chest X-ray at admission on ED was also 
assessed.

Specific treatment for COVID-19: the use of treatment against COVID (azithromycin, hydroxychloroquine, lopinavir/ ritonavir, corticosteroids, interferon, and immune modulator) was additionally reported.

\section{Statistical analysis}

Quantitative variables were expressed as means and standard deviations and qualitative variables as percentages. Differences in the quantitative variables between those who died and those who did not were assessed using the ANOVA or Kruskall Wallis test; differences in percentages were assessed using the chi squared test.

To assess the association between hospital mortality and each studied variable, we used multivariate logistic regression (results presented as odds ratios and their confidence intervals). With this purpose, 5 models were fitted. Model 1 was adjusted for sex and age. Next, the potential mediation of function (Barthel index) was assessed by adding to model 1 (model 2). Then, the score on Cumulative Illness Rating Scale- Geriatrics (CIRS-G) was added to the previous model (model 3). Further, a fourth model was created adjusted additionally for the presence of polypharmacy. Finally, model 5 was defined as model 4 plus adjusted for C-reactive protein.

The level of significance was set at $p$ value $<0.05$ or less. Statistical analyses were performed with STATA (version 14.0; Stata Corp., College Station).

\section{Figure 1. Flow chart of the participants included in the study}

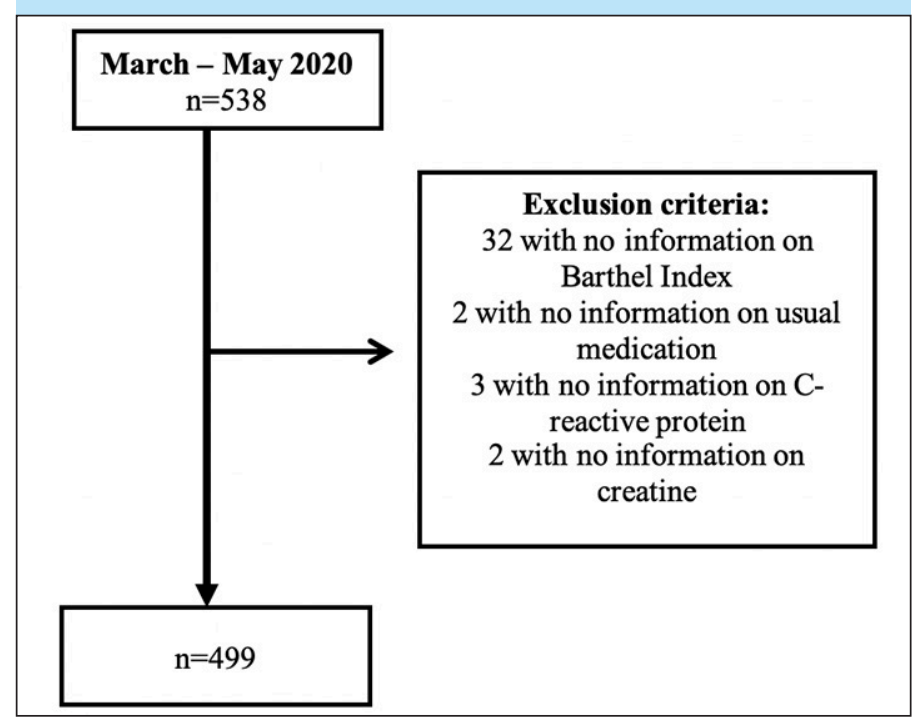

\section{Results}

\section{Differences between groups}

\section{Characteristics of study participants}

Population characteristics are described in Table 1. 499 participants with a mean age of $86.7 \pm 4.4$ (ranging from 80 to 106 years old) with a mean length stay of 12.8 days ( \pm 10.6$)$ were included in this study. In those who survived, the mean length stay was $15.1( \pm 10.3)$ days versus $8.7( \pm 10.1)$ in the deceased group $(\mathrm{p}=0.86)$.

The hospital mortality in our study was $37 \%$. Compared to the 319 survivors, the 187 non-survivors were predominantly men; with lower Barthel Index score; higher score in comorbidities indices (both CIRS-G and Charlson index); higher prevalence of diabetes, dementia, and cerebrovascular disease; and higher rates of hyperpolypharmacy, being all these differences statistically significant (table 1).

\section{Clinical Presentation, Laboratory and Imaging Findings}

Table 2 summarizes the initial symptoms, laboratory and imaging findings, and treatment received. According to clinical presentation, differences between the 2 groups were statistically significant for fever ( $73.4 \%$ non-survivors vs $62.9 \%$ survivors; $\mathrm{P}=0.02$ ), dyspnea (64.7 non-survivors vs $49.8 \%$ survivors; $\mathrm{P}=0.001)$ and mean oxygen saturation $(88.7 \% \pm 8.5$ nonsurvivors vs $93.3 \% \pm 5.0 ; \mathrm{P}=<0.001)$.

Regarding to laboratory findings (Table 2) compared to those who survived, people who died during hospitalization had higher rates of hypo or hypernatremia and lymphopenia. Additionally, an increase on inflammatory biomarkers was observed in non-survivors in CRP, procalcitonin, ferritin, and $\mathrm{d}$-dimer, all of them with p-value $<0.001$. Participants who died had also an increased glucose level and worse renal function.

According to chest X-ray, a normal image was more frequent in those who survived.

\section{Treatment}

Different treatment options related to COVID-19 were administrated during hospitalization (Table 2). The most used treatment regimen were hydroxychloroquine $(65.9 \%)$, corticosteroids $(28.5 \%)$, lopinavir/ritonavir (26.1\%), and azithromycin $(25.5 \%)$. Interferon and other immune modulator were rarely used. Compared to survivors, nonsurvivors received higher rates of azithromycin $(22.2 \%$ vs $31 \%$, respectively; $\mathrm{p}=0.03$ ) and lower rates of hydroxychloroquine (71.4\% vs $56.5 \%$, respectively; $\mathrm{p}=0.001$ ).

\section{Risk assessment of death}

Sociodemographic variables, functional status,

We assessed the risk factors associated to death through five models. Table 3 shows that, compared to female, being male was associated with a higher risk of hospital mortality due to covid-19, with an OR 1.50 (95\%CI 1.01-2.24, $\mathrm{p}=0.05)$. Additionally, each 5-points increase on Barthel Index [OR $1.01(95 \% \mathrm{CI} 1.00-1.02, \mathrm{p}=0.03)]$, as well as a higher Charlson Index score [OR 1.13 (95\%CI 1.02-1.26, $\mathrm{p}=0.03)$ ] and a higher number of comorbidities [OR $1.28(95 \% \mathrm{CI} 1.06-1.53, \mathrm{p}=0.01)$ ] 
Table 2. Symptoms, laboratory findings, imaging, treatment, and hospital mortality in old patients hospitalized by COVID-19 $[\mathrm{n}=499]$

\begin{tabular}{|c|c|c|c|c|}
\hline & Total & Recovered (n=319; 63\%) & Death $(n=187 ; 37 \%)$ & $P$ value \\
\hline \multicolumn{5}{|l|}{ Initial symptoms } \\
\hline Fever; n $(\%)$ & $333(66.7)$ & $198(62.9)$ & $135(73.4)$ & 0.02 \\
\hline Dyspnea; n (\%) & $276(55.3)$ & $157(49.8)$ & $119(64.7)$ & 0.001 \\
\hline Saturation (\%); mean (SD) & $91.6(6.8)$ & $93.3(5.0)$ & $88.7(8.5)$ & $<0.001$ \\
\hline Myalgias/arthralgia; n (\%) & $72(14.4)$ & $52(16.5)$ & $20(10.9)$ & 0.08 \\
\hline Headache; n (\%) & $23(4.6)$ & $19(6.0)$ & $4(2.2)$ & 0.05 \\
\hline Digestive symptoms; n (\%) & $115(23.1)$ & $81(25.7)$ & $34(18.5)$ & 0.06 \\
\hline Dizziness; n (\%) & $34(6.8)$ & $19(6.0)$ & $15(8.2)$ & 0.36 \\
\hline Normonatremia (135-145 mmol/L) & $352(70.5)$ & $236(74.9)$ & $116(63.0)$ & \\
\hline Hyponatremia (<135 mmol/L) & $125(25.1)$ & $71(22.5)$ & $54(29.4)$ & \\
\hline Hypernatremia (>145 mmol/L) & $22(4.4)$ & $8(2.5)$ & $14(7.6)$ & \\
\hline Lymphocytes (U/uL); mean (SD) & $1,008.6(1,739.9)$ & $1,068.0(2,061.2)$ & $903.8(931.4)$ & $<0.001$ \\
\hline Platelets (U/uL); mean (SD) & $192,803.7(91,952.6)$ & $201,243.6(98,530.8)$ & $178,262.5(77,596.7)$ & $<0.001$ \\
\hline C-reactive protein $(\mathrm{mg} / \mathrm{dL})$; mean $(\mathrm{SD})$ & $9.4(8.0)$ & $7.5(6.5)$ & $12.6(9.3)$ & $<0.001$ \\
\hline Procalcitonin (ng/mL); mean (SD) & $0.9(6.7)$ & $0.4(1.7)$ & $1.9(11.1)$ & $<0.001$ \\
\hline Proteins (g/dL); mean (SD) & $5.6(1.5)$ & $5.5(1.7)$ & $5.8(1.3)$ & 0.05 \\
\hline Albumin (g/dL); mean (SD) & $3.0(0.9)$ & $2.9(1.0)$ & $3.1(0.7)$ & 0.03 \\
\hline Lung imaging & & & & 0.04 \\
\hline No pneumonia; n (\%) & $90(18.0)$ & $66(20.7)$ & $25(13.4)$ & \\
\hline Unilateral pneumonia; n (\%) & 198 (39.7) & $115(36.1)$ & $86(46.0)$ & \\
\hline Bilateral pneumonia; n (\%) & $211(42.3)$ & $138(43.3)$ & $76(40.6)$ & \\
\hline \multicolumn{5}{|l|}{ Treatment } \\
\hline Azithromycin; n (\%) & $127(25.5)$ & $70(22.2)$ & $57(31.0)$ & 0.03 \\
\hline Hydroxychloroquine; n (\%) & $329(65.9)$ & $225(71.4)$ & $104(56.5)$ & 0.001 \\
\hline Lopinavir/ ritonavir; n (\%) & $130(26.1)$ & $77(24.4)$ & $53(28.8)$ & 0.28 \\
\hline Corticosteroids; n (\%) & $142(28.5)$ & $87(27.6)$ & $55(30.0)$ & 0.58 \\
\hline Interferon; n (\%) & $27(5.4)$ & $7(2.2)$ & $20(10.9)$ & $<0.001$ \\
\hline Immune modulator; $\mathrm{n}(\%)$ & $5(1.0)$ & $4(1.3)$ & $1(0.5)$ & 0.43 \\
\hline
\end{tabular}

SD: Standard Deviation; \%: Percentage. *p-values for differences in means were obtained from ANOVA or Kruskall Wallis, as appropriate; while p-values for differences in proportions were obtained from chi squared tests. 
Table 3. Risk factors associated to in-hospital mortality in older people with covid-19 ( $\mathrm{n}=499$ )

\begin{tabular}{|c|c|c|c|c|c|c|c|c|c|c|}
\hline & \multicolumn{2}{|c|}{ Model 1} & \multicolumn{2}{|c|}{ Model 2} & \multicolumn{2}{|c|}{ Model 3} & \multicolumn{2}{|c|}{ Model 4} & \multicolumn{2}{|c|}{ Model 5} \\
\hline & OR $(95 \% \mathrm{CI})$ & p-value & OR $(95 \% \mathrm{CI})$ & p-value & OR (95\% CI) & p-value & OR (95\% CI) & p-value & OR $(95 \% \mathrm{CI})$ & p-value \\
\hline \multicolumn{11}{|c|}{ Sociodemographic variables, functional status, comorbidities, and polypharmacy } \\
\hline Gender (male) & $1.53(1.06-2.2)$ & 0.02 & $1.62(1.11-2.35)$ & 0.01 & $1.58(1.08-2.30)$ & 0.02 & $1.63(1.12-2.38)$ & 0.01 & $1.50(1.01-2.24)$ & 0.05 \\
\hline Living (nursing home) & $1.54(0.92-2.59)$ & 0.10 & $1.21(0.68-2.14)$ & 0.52 & $1.23(0.69-2.18)$ & 0.49 & $1.21(0.68-2.15)$ & 0.52 & $1.31(0.72-2.39)$ & 0.38 \\
\hline Barthel Index & $1.01(1.00-1.02)$ & 0.01 & - & - & $1.01(1.00-1.02)$ & 0.03 & $1.01(1.00-1.02)$ & 0.02 & $1.01(1.00-1.02)$ & 0.03 \\
\hline Charlson Index & $1.15(1.04-1.27)$ & 0.01 & $1.1(1.03-1.26)$ & 0.01 & $1.14(1.02-1.26)$ & 0.02 & $1.13(1.02-1.26)$ & 0.02 & $1.13(1.02-1.26)$ & 0.03 \\
\hline Comorbidities & $1.22(1.07-1.38)$ & 0.002 & $1.19(1.04-1.35)$ & 0.01 & $1.26(1.05-1.49)$ & 0.01 & $1.24(1.04-1.47)$ & 0.02 & $1.28(1.06-1.53)$ & 0.01 \\
\hline Dementia & $1.66(1.09-2.53)$ & 0.02 & $1.37(0.85-2.22)$ & 0.20 & $1.36(0.84-2.20)$ & 0.22 & $1.32(0.81-2.14)$ & 0.27 & $1.49(0.89-2.49)$ & 0.13 \\
\hline Cerebrovascular disease & $1.93(1.10-3.38)$ & 0.02 & $1.77(1.00-3.12)$ & 0.05 & $1.70(0.94-3.06)$ & 0.08 & $1.73(0.96-3.15)$ & 0.07 & $2.08(1.12-3.87)$ & 0.02 \\
\hline Diabetes mellitus & $1.54(1.03-2.29)$ & 0.03 & $1.59(1.07-2.37)$ & 0.02 & $1.55(1.03-2.34)$ & 0.04 & $1.50(0.99-2.27)$ & 0.05 & $1.30(0.90-2.16)$ & 0.13 \\
\hline \multicolumn{11}{|l|}{ Polypharmacy } \\
\hline$<5$ & Ref. & & Ref. & & Ref. & & Ref. & & Ref. & \\
\hline $5-9$ & $1.54(0.96-2.48)$ & 0.07 & $1.52(0.94-2.45)$ & 0.09 & $1.60(0.96-2.67)$ & 0.07 & $1.61(0.95-2.66)$ & 0.07 & $1.62(0.94-2.78)$ & 0.08 \\
\hline$\geq 10$ & $1.95(1.19-3.18)$ & 0.01 & $1.84(1.12-3.02)$ & 0.02 & $2.03(1.10-3.74)$ & 0.02 & $2.04(1.11-3.75)$ & 0.02 & $2.00(1.04-3.82)$ & 0.04 \\
\hline \multicolumn{11}{|l|}{ Symptoms } \\
\hline Fever & $1.63(1.09-2.43)$ & 0.02 & $1.68(1.12-2.52)$ & 0.01 & $1.69(1.13-2.54)$ & 0.01 & $1.69(1.12-2.55)$ & 0.01 & $1.36(0.89-2.10)$ & 0.16 \\
\hline Dyspnea & $1.86(1.27-2.71)$ & 0.001 & $1.78(1.22-2.60)$ & 0.003 & $1.77(1.21-2.59)$ & 0.003 & $1.76(1.20-2.58)$ & 0.004 & $1.46(0.97-2.18)$ & 0.07 \\
\hline Oxygen level & $0.90(0.87-0.93)$ & $<0.001$ & $0.90(0.86-0.93)$ & $<0.001$ & $0.90(0.87-0.93)$ & $<0.001$ & $0.90(0.87-0.93)$ & $<0.001$ & $0.92(0.89-0.95)$ & $<0.001$ \\
\hline \multicolumn{11}{|l|}{ Radiology } \\
\hline No pneumonia & Ref. & Ref. & Ref. & Ref. & Ref. & & & & & \\
\hline Unilateral pneumonia & $1.86(1.08-3.20)$ & 0.03 & $2.01(1.18-3.59)$ & 0.01 & $2.21(1.25-3.89)$ & 0.01 & $2.28(1.29-4.03)$ & 0.01 & $1.83(1.01-3.30)$ & 0.05 \\
\hline Bilateral pneumonia & $1.32(0.77-2.29)$ & 0.31 & $1.48(0.85-2.59)$ & 0.17 & $1.53(0.87-2.68)$ & 0.10 & $1.51(0.86-2.65)$ & 0.15 & $1.16(0.65-2.09)$ & 0.61 \\
\hline \multicolumn{11}{|l|}{ Laboratory parameters } \\
\hline \multicolumn{11}{|l|}{ Sodium (mmol/L) } \\
\hline Normal values $(135-145)$ & Ref. & Ref. & Ref. & Ref. & Ref. & & & & & \\
\hline Hyponatremia & $1.58(1.04-2.41)$ & 0.03 & $1.61(1.06-2.47)$ & 0.03 & $1.61(1.05-2.46)$ & 0.03 & $1.56(1.01-2.39)$ & 0.04 & $1.36(0.86-2.11)$ & 0.19 \\
\hline Hypernatremia & $3.29(1.33-8.18)$ & 0.01 & $2.60(1.01-6.65)$ & 0.05 & $2.57(1.00-6.58)$ & 0.05 & $2.41(0.93-6.25)$ & 0.07 & $2.16(0.81-5.79)$ & 0.12 \\
\hline C-reactive protein $(\mathrm{mg} / \mathrm{dL})$ & $1.09(1.06-1.11)$ & $<0.001$ & $1.09(1.06-1.12)$ & $<0.001$ & $1.09(1.06-1.12)$ & $<0.001$ & $1.09(1.06-1.12)$ & $<0.001$ & - & - \\
\hline Creatine (mg/dL) & $1.58(1.25-2.00)$ & $<0.001$ & $1.58(1.25-1.99)$ & $<0.001$ & $1.57(1.24-1.99)$ & $<0.001$ & $1.62(1.27-2.05)$ & $<0.001$ & $1.48(1.15-1.89)$ & 0.002 \\
\hline Lymphocytes (U/uL) & $1.00(0.99-1.00)$ & 0.43 & $1.00(0.99-1.00)$ & 0.37 & $1.00(0.99-1.00)$ & 0.37 & $1.00(0.99-1.00)$ & 0.41 & $1.00(0.99-1.00)$ & 0.72 \\
\hline
\end{tabular}

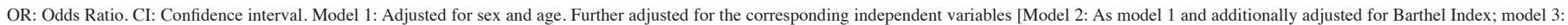

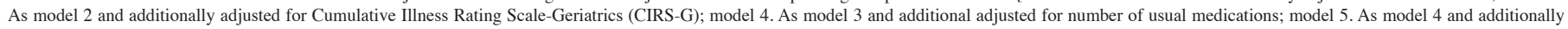
adjusted for C-reactive protein $(\mathrm{mg} / \mathrm{dL})]$

were related to a higher likelihood of mortality. Participants diagnosed by cerebrovascular disease were at higher risk of dying, compared to those who did not [OR 2.08 (95\%CI 1.12$3.87, \mathrm{p}=0.02)]$. Hyperpolypharmacy, but not polypharmacy was also linked to a higher rate of hospital mortality [OR 2.00 (95\% CI 1.04-3.82, $\mathrm{p}=0.04)]$.

No association was observed between place of living and risk of mortality.

\section{Clinical Presentation, Laboratory and Imaging Findings}

Higher oxygen levels were protective factor of mortality [OR $0.92(95 \%$ CI $0.89-0.95, \mathrm{p}<0.001)]$, compared to lower rates of oxygen. Moreover, compared to those with no pneumonia, only unilateral pneumonia showed an association with hospital mortality [OR 1.83 (95\% CI 1.01-3.30, $\mathrm{p}=0.05)$ ], whereas no relationship was observed with bilateral pneumonia.

Regarding to laboratory parameters, individuals with higher levels of CRP [OR 1.09 (95\%CI 1.06-1.12, p<0.001)] and creatine [OR $1.48(95 \%$ CI $1.15-1.89, \mathrm{p}=0.002)]$ were at higher risk of mortality, compared to those with lower values. Ferritin, procalcitonin, albumin, protein, cholesterol, and d-dimer levels were excluded from analysis due to missing values.

No association was observed between any type of treatment and risk of mortality.

\section{Discussion}

Our study showed that being male, having a worse functional status, a greater burden due to comorbidities, hyperpolipharmacy, being diagnosed of unilateral pneumonia, and having high levels of creatine and CRP at admission are independent risk factors for hospital mortality for people older than 80 with COVID-19. Additionally, higher levels of blood oxygen have a protective role for mortality in these patients.

In this analysis, male gender has been demonstrated to be a risk factor of mortality, being consistent with previous literature. A study published in 2020 reported that the angiotensin-converting enzyme 2 (ACE2) enzyme may be 
the host receptor for SARS-COV2, and this protein has a high expression in the lung tissues of men (15). Moreover, some previous studies have proposed a relation between that susceptibility and a X-linked inheritance, since both the androgen receptor gene and ACE2 genes are located at chromosome $\mathrm{X}(16,17)$, playing an important role in the innate and adaptive immunity of female patients.

We confirm the role of functional status in predicting mortality in this very old population. According to previous studies $(3,18-20)$, functional status was a strong predictor of risk of death in very old people with COVID-19 disease, showing an increase of $1 \%$ in the risk of mortality by each 5-points decrease. Compare to a previous Spanish study (3), although this percentage is lower than the one they observed (a mean increase of 10-15\% in the risk of death for each decrease of 5 points in Barthel Index). This lower effect of function can be explained by the higher age of the population in our study, taking into account that as people get older, the burden of age among mortality is the highest (21).

It is widely known the role of polypharmacy as a risk factor for adverse outcomes in older population (22-25), although this association is controversial in patients with COVID-19. In the study of Laosa et al (3), they did not find a role for polypharmacy (defined by taking $\geq 5$ ) in the full-adjusted model. However, similarly, in our study we only found an association with the risk of death when the number of usual drugs were 10 or higher, but not in those taking 5-10 drugs. These findings suggest that polypharmacy is a risk factor in these populations when it is remarkably high.

Severe COVID-19 disease is defined as dyspnea or blood oxygen saturation of $93 \%$ or less, among other clinical and radiological characteristics (4). As we described before, fever, dyspnea and desaturation were the most common symptoms in our sample. The three of them were also associated with increased risk of death, acting only the oxygen level as an independent risk factor of death. In other studies, the prevalence of cardinal symptoms such as fever and dyspnea appear to be similar in older patients (19). Desaturation and tachypnea have particularly been identified as common risk factors for advanced respiratory support and mortality (26). In our study patients with unilateral pneumonia were at higher risk of mortality compared to those without pneumonia but, contrary to what was expected, having bilateral pneumonia did not increase the risk of death. This finding must be taken with caution, as we only included the first chest radiography performed at admission. It is not uncommon for patients with COVID-19 disease to worsen in the following days from admission, developing an inflammatory response with the subsequent progression of the baseline infiltrates. In addition to it, the extension of the pulmonary infiltrates was not taken into account. Thus, mild, or dubious infiltrates in both lung fields were also named as bilateral pneumonia, even though the severity of those infiltrates were low.

When analyzing some biochemical parameters, higher levels of $\mathrm{C}$-reactive protein and creatine could be considered risk factors of mortality, as it has been demonstrated in previous studies $(26,27)$. These findings show up the association between an excessive systemic inflammatory response and a higher risk of poor outcomes, as well as the risk of mortality that lower estimated glomerular filtration rate involves. In fact, there is evidence in previous studies that chronic kidney disease contributes to death and it is associated with disease severity $(28,29)$. Hyponatremia and hypernatremia were also associated with a higher risk of death in almost all of our models. Dysnatremia has been related to a poor prognosis in hospitalized patients, specifically in those with communityacquired pneumonia (30-32). Additionally, a retrospective study conducted on 4664 patients aged 17 and older with COVID-19 infection (HOPE-COVID-19 study) (33), also demonstrated that both hyponatremia and hypernatremia were independently associated to in-hospital mortality.

Our study has several strengths and limitations. Among the first ones, this present work is unique by focusing on the oldest-old patients with a large sample size (499 subjects) composed by consecutively admitted, thus limiting the potential for selection bias. In addition, we enrolled a heterogeneous group with many different features and clinical presentations, allowing us to identify a variety of risk factors for severe COVID-19 in this population.

On the other hand, one potential limitation of our study is related to the period of time in which it was performed. Between the months of March and May, 2020, the Spanish health care system was close to collapse and we cannot discard that other very old patients with severe disease were not sent to an emergency room/hospital, including those with mild symptoms fit enough to home isolation without medical care or those with very poor prognosis living in nursing homes to whom only palliative care could be offered. Thus, some results in our study may be biased.

In conclusion, the current study showed that gender, functional status, and some clinical, laboratory, and radiology variables play an important role as an independent risk factors for hospital mortality in patients over 80 with COVID-19, providing useful information for an early identification of higher risk patients. The knowledge accumulated so far on how to treat hospitalized patients with COVID-19 should be used to improve survival of the most vulnerable individuals.

Declaration of conflict of interest: None.

Declaration of sources of funding: Fundación Fernández-Cruz.

Author Contributions: Study concept and design: all authors. Data collection: MRM, LFF, MMH, ACC, RSP. Statistical analyses: IRS; Draft of the manuscript: IRS, MRM, LFF, MMH, ACC, RSP. Full access to all of the data in the study and responsibility for the integrity of the data and the accuracy of the data analysis: IRS. Study supervision: PGG All authors reviewed the manuscript for important intellectual content and approved the final version.

Ethical standards: We consciously assure that for the manuscript «Functional, clinical, and sociodemographic risk variables of hospital mortality by COVID-19 in people ove 80 years old" for the following is fulfilled: • This manuscript is the authors' own original work, which has not been previously published elsewhere, whole or in part. - The paper is not currently being considered for publication elsewhere. If it will be accepted, the paper will not be published elsewhere in the same form without the written consent of the copyright holder. $\bullet$ All sources and previous publications used are properly cited, with proper reference. $\bullet$ All co-authors are included on the paper, and all of them have approved the final version of the manuscript and have agreed that the work is ready for submission. - All authors will take public responsibility for the manuscript content. - All authors meet the criteria for authorship stated in the niform Requirements for Manuscripts Submitted to Biomedical Journals. 


\section{References}

1. Wang C, Horby PW, Hayden FG, Gao GF. A novel coronavirus outbreak of global health concern. Lancet 2020;395:470-473.

2. WHO Coronavirus (COVID-19) Dashboard I WHO Coronavirus (COVID-19) Dashboard With Vaccination Data Available at: https://covid19.who.int/. Accessed May 6, 2021.

3. Laosa O, Pedraza L, Álvarez-Bustos A, Carnicero JA, Rodriguez-Artalejo F, Rodriguez-Mañas L. Rapid Assessment at Hospital Admission of Mortality Risk From COVID-19: The Role of Functional Status. J Am Med Dir Assoc 2020;21:1798-1802. e2.

4. Vrillon A, Hourregue C, Azuar J, Grosset L, Boutelier A, Tan S, Roger M, Mourman V, Mouly S, Sène D, François V, Dumurgier J, Paquet C. COVID-19 in Older Adults: A Series of 76 Patients Aged 85 Years and Older with COVID-19. J Am Geriatr Soc 2020;68:2735-2743.

5. COVID-19 strategy update - 14 April 2020 Available at: https://www.who.int/ publications/i/item/covid-19-strategy-update---14-april-2020. Accessed May 6, 2021

6. Redondo-Bravo L, Moros MJS, Sanchez EVM, et al. The first wave of the COVID-19 pandemic in Spain: Characterisation of cases and risk factors for severe outcomes, as at 27 April 2020. Eurosurveillance 2020;25:2001431.

7. Defunciones por covid-19 virus identificado y virus no identificado (sospechoso), mes de defunción, sexo y edad Available at: https://ine.es/jaxi/Tabla.htm?path=/t15/p417/ covid/10/\&file=01001.px\&L=0. Accessed May 6, 2021 .

8. Huang C, Wang Y, Li X, et al. Clinical features of patients infected with 2019 novel coronavirus in Wuhan, China. Lancet 2020;395:497-506.

9. Wang L, He W, Yu X, Hu D, Bao M, Liu H, Zhou J, Jiang H. Coronavirus disease 2019 in elderly patients: Characteristics and prognostic factors based on 4-week follow-up. J Infect 2020;80:639-645.

10. Liu K, Chen Y, Lin R, Han K. Clinical features of COVID-19 in elderly patients: A comparison with young and middle-aged patients. J Infect 2020;80:e14-e18.

11. Richardson S, Hirsch JS, Narasimhan M, et al. Presenting Characteristics, Comorbidities, and Outcomes among 5700 Patients Hospitalized with COVID-19 in the New York City Area. JAMA - J Am Med Assoc 2020;323:2052-2059.

12. Huang J, Cheng A, Kumar R, Fang Y, Chen G, Zhu Y, Lin S. Hypoalbuminemia predicts the outcome of COVID-19 independent of age and co-morbidity. J Med Virol 2020;92:2152-2158.

13. Hoogendijk EO, Romero L, Sánchez-Jurado PM, Flores Ruano T, Viña J, RodríguezMañas L, Abizanda P. A New Functional Classification Based on Frailty and Disability Stratifies the Risk for Mortality Among Older Adults: The FRADEA Study. J Am Med Dir Assoc 2019;20:1105-1110.

14. Hewitt J, Carter B, Vilches-Moraga A, et al. The effect of frailty on survival in patients with COVID-19 (COPE): a multicentre, European, observational cohort study. Lancet Public Heal 2020;5:e444-e451.

15. Li R, Qiao S, Zhang G. Analysis of angiotensin-converting enzyme 2 (ACE2) from different species sheds some light on cross-species receptor usage of a novel coronavirus 2019-nCoV. J Infect 2020;80:469-496.

16. Wambier CG, Goren A, Vaño-Galván S, Ramos PM, Ossimetha A, Nau G, Herrera S, McCoy J. Androgen sensitivity gateway to COVID-19 disease severity. Drug Dev Res 2020;81:771-776.
17. Chen N, Zhou M, Dong X, Qu J, Gong F, Han Y, Qiu Y, Wang J, Liu Y, Wei Y, Xia J, Yu T, Zhang X, Zhang L. Epidemiological and clinical characteristics of 99 cases of 2019 novel coronavirus pneumonia in Wuhan, China: a descriptive study. Lancet 2020;395:507-513.

18. Alsahab M, Beishon L, Brown B, et al. Age and frailty are independently associated with increased COVID-19 mortality and increased care needs in survivors: results of an international multi-centre study. Age Ageing 2021;50:617-630.

19. Knopp P, Miles A, Webb TE, Mcloughlin BC, Mannan I, Raja N, Wan B, Davis D. Presenting features of COVID-19 in older people: relationships with frailty, inflammation and mortality. Eur Geriatr Med 2020;11:1089-1094.

20. Blomaard LC, van der Linden CMJ, van der Bol JM, et al. Frailty is associated with in-hospital mortality in older hospitalised COVID-19 patients in the Netherlands: the COVID-OLD study. Age Ageing, 2021. doi:10.1093/ageing/afab018.

21. Sourial N, Bergman H, Karunananthan S, Wolfson C, Payette H, Gutierrez-Robledo LM, Béland F, Fletcher JD, Guralnik J. Implementing frailty into clinical practice: A cautionary tale. Journals Gerontol - Ser A Biol Sci Med Sci 2013;68:1505-1511.

22. Midão L, Brochado P, Almada M, Duarte M, Paúl C, Costa E. Frailty status and polypharmacy predict all-cause mortality in community dwelling older adults in Europe. Int J Environ Res Public Health 2021;18. doi:10.3390/ijerph18073580.

23. Chen N, Alam AB, Lutsey PL, MacLehose RF, Claxton JS, Chen LY, Chamberlain AM, Alonso A. Polypharmacy, Adverse Outcomes, and Treatment Effectiveness in Patients $\geq 75$ With Atrial Fibrillation. J Am Heart Assoc 2020;9:e015089.

24. Maher RL, Hanlon J, Hajjar ER. Clinical consequences of polypharmacy in elderly. Expert Opin Drug Saf 2014;13:57-65.

25. Sabzwari SR, Qidwai W, Bhanji S. Polypharmacy in elderly: A cautious trail to tread. J Pak Med Assoc 2013;63:624-627.

26. Lee JY, Kim HA, Huh K, Hyun M, Rhee JY, Jang S, Kim JY, Peck KR, Chang HH Risk factors for mortality and respiratory support in elderly patients hospitalized with COVID-19 in Korea. J Korean Med Sci 2020;35. doi:10.3346/JKMS.2020.35.E223.

27. Berenguer J, Ryan P, Rodríguez-baño J, Jarrín I. Characteristics and predictors of death among 4035 consecutively hospitalized patients with COVID-19 in Spain.

28. Fang X, Li S, Yu H, Wang P, Zhang Y, Chen Z, Li Y, Cheng L, Li W, Jia H, Ma X. Epidemiological, comorbidity factors with severity and prognosis. 2020;12:1249312503.

29. Zhang G, Hu C, Luo L, Fang F, Chen Y, Li J, Peng Z, Pan H. Clinical features and short-term outcomes of 221 patients with COVID-19 in Wuhan, China. J Clin Virol 2020;127. doi:10.1016/j.jcv.2020.104364.

30. Waikar SS, Mount DB, Curhan GC. Mortality after Hospitalization with Mild, Moderate, and Severe Hyponatremia. Am J Med 2009;122:857-865.

31. Bataille S, Baralla C, Torro D, Buffat C, Berland Y, Alazia M, Loundou A, Michelet P, Vacher-Coponat $\mathrm{H}$. Undercorrection of hypernatremia is frequent and associated with mortality. BMC Nephrol 2014;15:1-9.

32. Nair V, Niederman MS, Masani N, Fishbane S. Hyponatremia in community-acquired pneumonia. Am J Nephrol 2007;27:184-190.

33. Ruiz-Sánchez JG, Núñez-Gil IJ, Cuesta M, et al. Prognostic Impact of Hyponatremia and Hypernatremia in COVID-19 Pneumonia. A HOPE-COVID-19 (Health Outcome Predictive Evaluation for COVID-19) Registry Analysis. Front Endocrinol (Lausanne) 2020;11:1-12.

How to cite this article: I. Rodríguez-Sánchez, M. Redondo-Martín, L. FuronesFernández et al. Functional, Clinical, and Sociodemographic Variables Associated with Risk of In-Hospital Mortality by COVID-19 in People over 80 Years Old. J Nutr Health Aging. 2021;25(8):964-970; http://dx.doi.org/10.1007/s12603-021-1664-9 